indices underestimate biologic activity and cannot detect transmural disease. We aimed to assess the role of CT enterography (CTE) in assessing Crohn's disease (CD).

Methods A retrospective review of 406 consecutive CTE studies was performed between January 2009 and December 2012 at our institution. Clinical data including demographics, disease characteristics and therapy were obtained from electronic patient record review. Inflammatory markers, radiological investigations and ileocolonoscopy when performed within 90 days of CTE were recorded. CTE reports were recorded using accepted activity criteria- small bowel dilatation, stenosis, wall thickening, enhancement, mucosal irregularity, mesenteric inflammation, hypervascularity, lymph node enlargement, abscesses, fistulation and extraintestinal features.

Results Of 175 patients with IBD at time of CTE, 154 had CD. Ninety-four of 154 patients were female, mean age 52 (range 16-87) and median term of follow up of 5 years (range 0-35).

Abnormalities were noted in 100 scans; 56 had active non-stricturing, 42 active stricturing and 2 fibrostenotic disease. Within active groups, there were 10 fistulae and 3 abscesses in 11 patients. Ileocolonoscopy was performed in 42 patients with 27 showing active inflammation and raised CRP in 38/96. Treatment was increased in $52 \%$ of the active non-stricturing group, $6 / 29$ to azathioprine, 4/29 to biologics, $4 / 29$ to methotrexate, $7 / 29$ to steroids, $5 / 29$ to surgery with no change in the remaining $48 \%$, of whom $8 / 12$ had inactive disease at ileo-colonoscopy and 16/24 normal CRP.

In $57 \%$ of active stricturing patient treatment was increased in 1 to azathioprine, 11 to biologics, 5 to surgery. Twelve of 26 patients in this group had an elevated CRP and 10/13 had active colitis at ileo-colonoscopy.

Of 53 normal CTE, treatment was escalated in 3 to methotrexate or azathioprine with colitis at colonoscopy and unchanged in $92 \%$. Conclusion Concerns regarding potential cumulative effects of ionising radiation are valid but likely to be offset by changing technology and reduction in average doses of radiation.

CTE has a role in well-selected patients with CD (e.g. age $>50$, very sick patients) identifying active disease and influencing meaningful therapeutic decisions.

Disclosure of Interest None Declared.

\section{PWE-086 THE ROLE OF HUMAN NEUTROPHIL ELASTASE AND ITS INHIBITOR ELAFIN IN ULCERATIVE COLITIS}

doi:10.1136/gutjnl-2013-304907.374

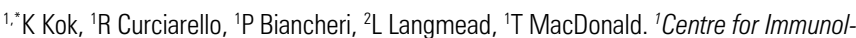
ogy and Infectious Disease, Barts and The London School of Medicine and Dentistry; ${ }^{2}$ Royal London Hospital, London, UK

Introduction Mucosal inflammation in ulcerative colitis (UC) is characterised by an influx of neutrophils which secrete large amounts of human neutrophil elastase (HNE), causing matrix degradation. They also produce the elastase-specific inhibitor, elafin. The aim of this study is to evaluate the relative production of elastase and elafin in active UC, and to investigate the modulatory effect of elafin on mucosal proteolytic activity ex vivo.

Methods We utilised intestinal biopsies from 18 patients with active UC and 12 non-UC healthy controls. Biopsies were homogenised and lysed to extract mucosal proteins. Proteolytic activity, using elastin as a substrate, was determined. Concentrations of elafin were measured using ELISA. The effect of protease inhibitors on proteolytic activity were determined in vitro using elafin, marimastat (matrix metalloproteinase inhibitor) and the synthetic elastase inhibitor, AAPV [N-(Methoxysuccinyl)-Ala-AlaPro-Val Chloromethyl Ketone]. The effect of elafin on proteolytic activity ex vivo was assessed by 24 hour organ culture in the presence and absence of elafin. Unpaired Student's t-test was used for statistical analyses.
Results Mucosal protein homogenates from patients with active UC displayed higher proteolytic activity in comparison to healthy controls $(p=0.002)$. Elafin levels were increased in mucosal homogenates from active UC ( $p=0.007)$. The addition of elafin, marimastat or AAPV, in vitro, each diminished proteolytic activity. Organ culture of UC biopsies in the presence of elafin reduces the proteolytic activity of active UC ex vivo (n.s.).

Conclusion Colonic mucosal tissue from UC patients displays significantly higher elastinolytic activity in comparison to healthy controls. The addition of elafin has a restorative effect on the elastinolytic activity of UC mucosal homogenates, with the most notable effect in those tissues that had highest proteolytic activity. This occurs in the presence of significantly higher quantities of elafin in active UC mucosa. These data also show a beneficial modulatory effect of elafin on human gut tissue, suggesting a possible role for supplementary elafin in the treatment of UC.

\section{Disclosure of Interest None Declared.}

\section{PWE-087 A CRITICAL REVIEW OF GROUNDED THEORY USE IN INFLAMMATORY BOWEL DISEASE STUDIES}

doi:10.1136/gutjnl-2013-304907.375

1, ${ }^{*} \mathrm{~K}$ C Fragkos, ${ }^{1} \mathrm{~A}$ Forbes. ${ }^{1}$ Centre for Gastroenterology and Nutrition, University College London, London, UK

Introduction Grounded Theory (GT) is a research methodology predominantly used with qualitative data. The purpose of the present study is to critically evaluate the use of GT in inflammatory bowel disease (IBD) studies and to examine the clinical implications this entails.

Methods A systematic literature review was performed using keywords Grounded Theory and IBD in Pubmed, EMBASE and Scopus with no time limits. Assessment of GT was performed using standard criteria suggested by Glaser (1998). The application of the following basic principles was examined: simultaneous data collection and analysis; construction of analytic codes and categories from data, not from preconceived logically deduced hypotheses; use of constant comparative method; advancement of theory development during each step of data collection and analysis; memo-writing; sampling aimed toward theory construction (theoretical); Literature review after the core category emergence. Results Fifteen studies have used GT investigating patient education, quality of life, experiences with therapeutic strategies or coping mechanisms in IBD, providing theories based on emerging categories. About half of all studies have applied the basic principles of GT, with the remaining studies being unclear or having not applied them. The most reported priniciple was Glaserian selective coding and least reported were memoing, theoretical sampling and the achievement of theoretical completeness, while the identification of the core category was unclear in many instances (Figure 1). These weaknesses are attributed predominantly to methodological, verification and reporting bias. These biases affect the applicability of these results in clinical practise. Hence, results concerning quality of life or experiences of IBD patients should be treated with caution, as they could represent authors' predisposition from the their experience (empirical or from literature reviews).

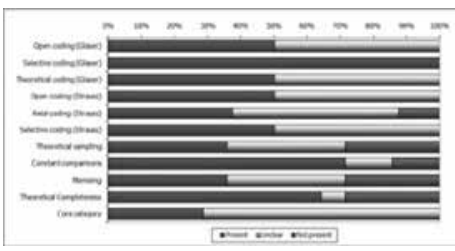

Abstract PWE-087 Figure 1 